\title{
Utilising Contextual Memory Retrieval Cues and the Ubiquity of the Cell Phone to Review Lifelogged Physiological Activities
}

\author{
Aiden R. Doherty ${ }^{1,2}$ and Kristin M. Tolle ${ }^{1}$ and Alan F. Smeaton ${ }^{2}$ \\ ${ }^{1}$ Microsoft Research Redmond, WA, USA \\ ${ }^{2}$ CLARITY: Centre for Sensor Web Technologies, Dublin City University, Ireland
}

\begin{abstract}
In today's healthcare world where we react to conditions that have already developed, lifelogging technologies may offer a glimpse into a future world of proactive healthcare where symptoms of conditions are detected at much earlier stages. At the end of last year it was estimated that there were 4 billion cell phones in the world, in comparison to just over 1 billon PCs. In this paper we discuss a framework, which leverages the ubiquity of the cell phone, to aggregate data from multiple wearable biological sensors. This physiological lifelogged data can then be queried via an interface which utilises contextual memory retrieval cues to assist people remember what type of activity they were doing at a particular time. This may be helpful in the diagnosis of potential medical conditions or encourage wellness behaviours.
\end{abstract}

\section{Categories and Subject Descriptors}

H.3.3 [Information Storage and Retrieval]: Information Search and Retrieval; H.5.0 [Information Interfaces and Presentation]: General; J.4.0 [Computer Applications]: Social and Behavioral Sciences

\section{General Terms}

Design, Human Factors, Management

\section{Keywords}

cell phone ubiquity; lifelogging; physiological user interfaces; sensor data aggregation

\section{INTRODUCTION}

Lifelogging is a term used to describe recording different aspects of your daily life, in digital form, for your own exclusive personal use. It is a form of reverse surveillance, sometimes termed sousveillance, referring to us - the subjects doing the watching, of ourselves [9]. Sensors for healthcare

Permission to make digital or hard copies of all or part of this work for personal or classroom use is granted without fee provided that copies are not made or distributed for profit or commercial advantage and that copies bear this notice and the full citation on the first page. To copy otherwise, to republish, to post on servers or to redistribute to lists, requires prior specific permission and/or a fee.

IMCE'09, October 23, 2009, Beijing, China

Copyright 2009 ACM 978-1-60558-758-5/09/10 ...\$10.00. monitoring have become fairly ubiquitous and as a result of this lots of "lifelogged" data can now be generated on a per user basis. As we are all individuals, our data is very individualised - we want health-based baseline information that is relevant to us. What appears to be an anomaly for one person can be the normal state for another. Even commonly assumed standards are highly non-representative of what represents "wellness" in an individual. Therefore being able to capture and query across our own data is a far more predictive and useful mechanism for determining a concerning condition or disease.

Cell/mobile phones are now ubiquitous with over 4 billion in the world today [27]. Without a doubt they are becoming the easily portable server capable of storing a myriad of the sensor data as well as transmit it to "the cloud" for long term storage. While it is not safe to assume that a $\mathrm{PC}$ will always be nearby, it is almost certainly reasonable to assume a cell phone will be. Cell phones are also a great mechanism for transmitting summarized information back to the userUin particular through low bandwidth mechanisms such as SMS.

To create the ability to analyse our data, being able to successfully query information across individually generated data streams from sensors is important. It is also important for the information to be presented to a user in a readily understandable manner. This can partly be accomplished by enabling users to query that information across the various sources without losing the ability to show they came from multiple data sources. At the end of the day, human eyes are the best way to evaluate and assimilate the information. Therefore it is important that we make the user interface a priority.

Ultimately the challenge we are attempting to address in this paper is how to store data from disparate physiological sources, enable visualization of these different data types, and finally enable consolidation and access through a single interface and allow for interrogation of the data by the user. A contribution of this work is an Open Source release which will be helpful for developers to visualise their own custom physiological sensor data or to health conscious end users who are trying to make sense of their own "lifelogged" physiological data.

\subsection{Scenario: Elevated heart rate while public speaking}

Sam is reviewing his heart rate monitor data for the last month in the SmartLogger UI. He notices an unusual spike that occurs with regular frequency (e.g., once a week). Sam highlights a particular region of the chart (implies the abil- 
ity to filter/limit the data to a particular week/day/hour) so that he can get a better idea of the context of the situation. He sees that it happens on Wednesday, so he selects one of those Wednesday's for review. On the refreshed chart he can see that the anomaly occurs during the team meeting that occurs weekly between $9 \mathrm{AM}$ and $11 \mathrm{AM}$ - not during the whole meeting, only during particular instances. Sam highlights the timeframe of the occurrences in the SmartLogger UI and right clicks to see what information he has in association with that timeframe. Fortunately, he had his cell phone on "automatic image capture" during the meeting. He discerns from the images that the spike during this particular meeting occurs when he is addressing the team (performing public speaking). He zooms out to select another Wednesday to confirm if his other spikes occur during similar instances. Not only can Sam view his heart-rate data, but he can also view other forms of captured data on the same graph. If he captured blood pressure, Sam could check if there is a correlation between his heart rate and blood pressure.

\subsection{Scenario: Sleep Apnoea}

Molly wakens up feeling tired every day. It has been suggested by her doctor that she may have obstructive sleep apnoea. Normally a patient would need to go to a sleep centre overnight to confirm the diagnosis. Her doctor thinks Molly is an excellent candidate to do a clinical trial of a new neck cuff sensor device for that very purpose. The neck cuff monitors blood oxygen, pulse, snoring and breath sounds (via microphone), head position (3d accelerometer) and galvanic skin resistance. This data is then captured by a smartphone resting near her bed for later uplink to the doctor's server for more analysis. Being able to monitor at home will give the doctor a more accurate reading since the patient is in familiar surroundings, thus disruption will not be related to other problems introduced by a foreign environment. Because the device is portable, data capture occurs over several nights. Molly is instructed to wear the device for a week and each morning to transfer the data from the cell phone to the doctor on mornings when she feels especially affected. She is to return to the office the following week for further information on her condition unless something more serious is indicated by early data. The doctor, running the SmartLogger software, browses Molly's data to look for anomalies. As the week progresses he can see patterns emerge at certain hours of the night that do not seem to be related to the disorder. He converses with Molly and discovers that these disruptions have to do with her partner's routine rather than something more serious. Molly is relieved and can now take actions to improve her sleeping conditions.

\subsection{Scenario: Adding a new type of sensor to SmartLogger}

Mary has just gotten back for the Consumer Electronics Show. While there she found out about a sensor she had never heard of before that monitors your "mood". She is already a SmartLogger developer and has added several other types of sensors to the SmartLogger Platform. So she is very familiar with doing this. She opens her application and puts in the functions to start having the data recorded in the SmartLogger system by providing the appropriate parameters, and she is now ready to integrate mood data from the device into his application. After compiling, she puts on the sensor and starts the application she built based on the SmartLogger platform. The SmartLogger application starts picking up the device's transmission and from now on SmartLogger tracks this information along with the other sensors she has previously set up. Later her SmartLogger desktop user interface automatically incorporates this new stream of information and presents it in a meaningful graphical manner.

\section{RELATED WORK}

Figure 1 attempts to convey the visitation pattern we have when going to our medical practitioners over our lifetime. In essence, when we visit our GP they only are able to make decisions based on the information we provide in that snapshot in time. The result of this is that it's very difficult to build up a complete picture of an individual's general wellbeing. During those visits to a GP, a patient is often asked on how they have been feeling in the past, and it is often difficult to remember what they have been doing.

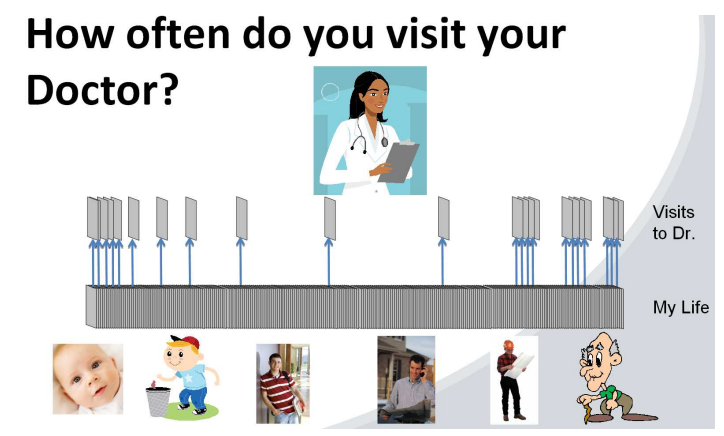

Figure 1: Our estimate on a typical visitation pattern to one's general practitioner

\subsection{Research on the Human Memory System}

As Schacter notes, while biological memory serves us well, it is highly selective and fallible [24]. We believe that to help individuals make better informed decisions regarding their healthcare, it will be important to understand how the human memory system operates and how it can also be assisted.

Atkinson and Shiffrin proposed a multi-store model of the human memory system in 1968 [2]. The model states that there are 3 distinct memory systems present in the human brain, namely one to deal with sensory inputs, one that relates to short-term memories, and finally a distinct system that deals with long-term memories only. Since 1968 more advanced, and complex, memory systems have been proposed, but for the purposes of this paper the Atkinson and Shiffrin model is sufficient. The sensory memory store only stores information for fractions of a second, and for each of the senses e.g. visual, audio, touch, smell, etc. Elsweiler notes that short-term memories are only retained for between 0.5 and 4 seconds, and he points to a number of theories ranging from "... decay - forgetting due to decay of unused information ... and interference - forgetting because of new information interfering with old information ...", but this debate remains unresolved [10].

What we are most interested in though is the long-term memory system which Anderson divides into declarative and 
procedural memories [1]. Elsweiler notes that procedural memories refer to remembering skills e.g. how to ride a bicycle, how to walk, how to swim, etc. [10]. Anderson further splits up declarative memories into semantic and episodic memories. Semantic memories are knowing about facts e.g. Paris is the capital of France, England won the soccer world cup in 1966, China is the most populous country in the world, etc. Episodic/autobiographical memories refer to those that are of personal experiences e.g. remembering when one's child first talks, recalling a conversation with one's friends from the previous evening, etc. Tulving describes that "...episodic memory does exactly what the other forms of memory do not and cannot do - it enables the individual to mentally travel back into her personal past ..." $[26]$.

One of the most important facets related to the autobiographical memory system, in terms of technology research, is the evidence pointing towards "cued recall" leading to retrieval of memories that can't be retrieved via "free recall". Purdy et. al. state that "... scores on recognition tests are usually much higher than scores based on recall ..." [22]. This indicates that users prefer to be prompted with cues, rather than being asked to retrieve memories from scratch. Baddeley also echoes this point, stating that "... it is as if the cues direct you to search in the appropriate location in memory, and as such allow access to traces which would otherwise have been missed. While there is no doubt that both cueing and recognition can reveal information which is not accessible using straightforward unaided recall, it could also be argued that the memory trace is present but not strong enough to allow recall ..." [3]. In essence, good cues, which are related to how the brain encoded the memory in question, lead to strong retrieval performance.

\subsection{Lifelogging and Physiological Recording}

Naturally the idea of exploiting technology to aid memory has come into focus. Barreau states that '... Digital memories surpass biological memories by enabling people to directly see and interact with captured information. This powerful capability can help people manage their personal information ...' [5]. Indeed some recent visual lifelogging research using the SenseCam has shown much promise in providing cues to help individuals remember things that they otherwise may have forgotten $[13,6,8]$.

There has also been much research placed into the constant monitoring of physiological data sensors. An important ergonomical trait is to have just one local base to gather that data, such as a cell phone, PDA, or custom made data aggregation device. Jovanov and Zhang provide an overview on research in the field of personal health monitoring, where the issue of choice of wireless networking protocols has received much attention [16]. Indeed these "body area networks" (BANs) are often equipped with a choice of sensors recording heart rate, accelerometer, ECG, temperature, and blood pressure [19, 23, 21]. Due to the relative youth of this field, it is quite understandable that these efforts are all custom-made and are not easily available. Also in some instances the emphasis has been on monitoring the health of those who are already unwell [23, 25], rather than on monitoring the constant wellness of healthy people (which may help bring us closer to a goal of preventative healthcare).

We believe that constant wellness monitoring through the aggregation of data from physiological sensors should be made easily available to as many people as possible. Given the ubiquity of the cell phone [27], we believe that this platform should be used for such monitoring. Even though Bluetooth may not be the ideal wireless protocol in aggregating data from physiological sensors to the cell phone (in comparison to ZigBee for example [15]), we believe that it should currently be used purely due to the concentration of cell phones that have Bluetooth installed as standard [11]. Some projects have used the cell phone to monitor data via Bluetooth (e.g. [21, 23]), and in our work we follow this approach, while proposing an open framework that allows the easy addition and integration of additional sensors that transmit via Bluetooth.

Much of the prior work in this domain has concentrated on the considerable challenges of gathering this physiological in a non-intrusive manner. However we haven't found any literature concentrating on how lifelogged physiological data should be presented to users so that they can effectively review it. In most instances the data is presented in chart format e.g. [18]. However, given the characteristics of the human memory system which we have just highlighted, we believe that it is important to provide the users with contextual data to help them explain why they were in a certain physiological state. Also we want to provide users with easy-to-adjust temporal query abilities, which we will now discuss.

\section{SMARTLOGGER SYSTEM}

Figure 2 provides an overview of our "SmartLogger" System. To the left we convey the idea of a cell phone (of which there are approximately $4 \mathrm{Bn}$ such devices in the world) platform that is capable of reading data from multiple sources of data. This data is then stored on some central repository, which can then be viewed at a later stage via an intuitive interface.

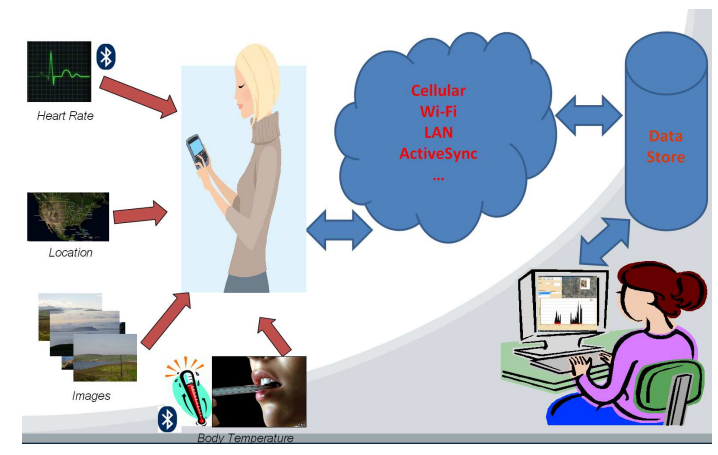

Figure 2: An overview of our "SmartLogger" system

Concentrating on the actual cell phone logger, we have developed a framework that is capable of easily being extended to include data from new sensors. Provided that these new physiological sensors can transmit their data via Bluetooth, a cell phone can be configured to read their outputs and store them as an XML file. One of the main purposes of this project is to encourage development of wearable sensing technologies and to make it easy for these developers to incorporate their sensors into a cell phone data logging platform. This means that there will be heterogeneous and incomplete data for the platform to deal with, and as a result it was decided to store the data in an XML format 
on the cell phone. This logger has been developed using the Windows Mobile C-sharp .NET framework, and runs on Windows Mobile cell phones.

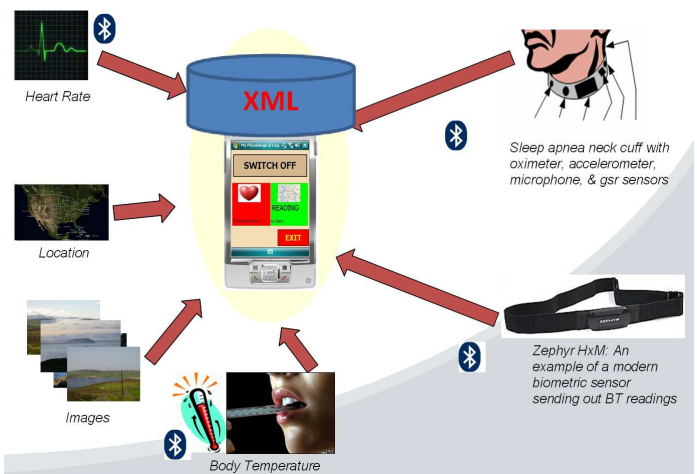

Figure 3: "SmartLogger" system on windows mobile devices

As motivated in prior sections, we wish to exploit certain characteristics of the human memory system to help users review their own physiological data. To achieve this we propose the interface in Figure 4 containing a query panel, a chart panel, and a context panel.

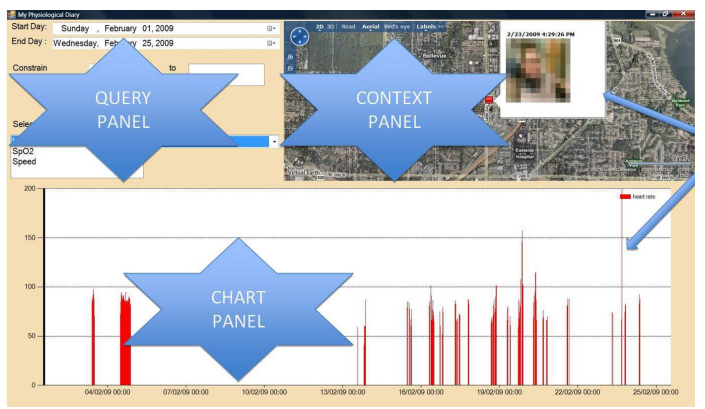

Figure 4: "My Physiological Diary" - an interface to assist people review data gathered by "SmartLogger"

The query panel allows users adaptively search for data based on time predominantly, thus exploiting the fact that humans have an acceptable memory of when events of interest temporally occur [17]. The chart panel provides users with a visual representation of their physiological activities, thus allowing users to immediately identify distinct events (potential anomalies) e.g. when a user's heart rate is unusually high or low. By clicking on any value of interest, the user can shift her/his attention to the context panel. This panel is very important in providing other contextual cues to the user to help provide additional memory cues to better explain the reasons for a certain physiological reading. Contextual cues can include the location the user was at, any images that the user may have captured at that time, any audio recorded (e.g. snoring and breathing sounds), etc.

As can be seen in Figure 5 the user can chart multiple (Min-Max normalised [20]) physiological values at the same time to visually inspect for any relationships between the values. Also using the query panel, the user can specify to "group graphs by" each day, thus helping them quickly identify daily patterns.

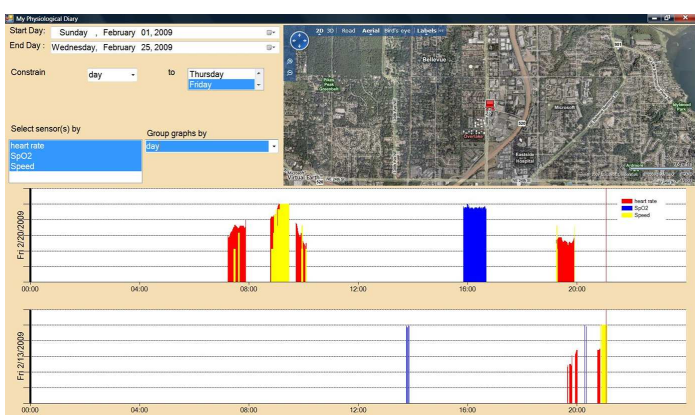

Figure 5: "My Physiological Diary" - identify daily trends across multiple data sources

\section{ANECDOTAL RESULTS}

To provide some anecdotal results on our system one of the authors gathered physiological data over a period of 3 weeks, at an average of 1 hour 45 minutes per day. We gathered location/GPS data ${ }^{1}$, heart rate data $^{2}$, and occasionally blood oximetry levels ${ }^{3}$.

Figure 6 displays our interface charting all of the heart rate data gathered over a 3 week time period. The use of visualising the data via a chart enables us to quickly identify anomaly events. One that obviously stands out is highlighted, where the user's heart rate was over $150 \mathrm{bpm}^{4}$. To help us determine possible reasons we double click on this value to display the associated information in the context panel. We can then immediately explain the reason for this unusually high heart rate value since the location context tells us that our user was working out at a health club. This shows the value of having contextual information to explain outlier events.

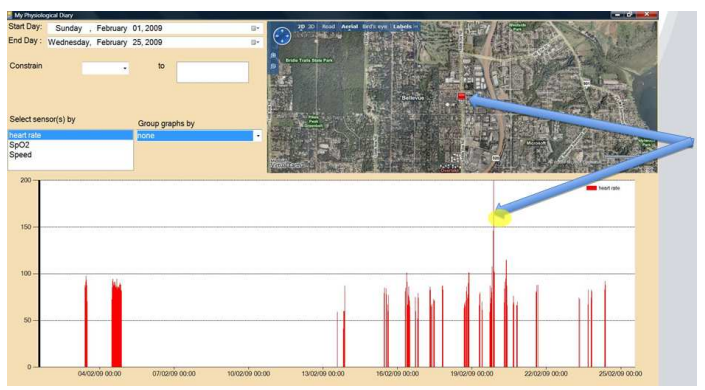

Figure 6: Anecdotal result - location context helping explain outlier events

Figure 7 again displays our interface plotting all the heart rate data gathered over a 3 week time period. This time we are interested in looking at an event where the heart rate appears normal before an elevation in the heart rate values. By double clicking on the relevant value on the chart, the user can see they were at work, and in addition to this an image is provided to provide further contextual information. By also inspecting the exact time of the event $(4.30 \mathrm{pm})$ we can then see that the later activity is likely to be the user

\footnotetext{
${ }^{1}$ via Windows Mobile HTC cell phone

${ }^{2}$ via ZephyrTech HxM device

${ }^{3}$ via Nonin SpO2 reader

${ }^{4}$ heart beats per minute
} 
walking home from work (which was then confirmed by clicking on the elevated heart rate activity to see the associated location data). Again we see how the introduction of contextual information (not only location, but also images plus time) helps to explain the physiological values associated with numerous activities.

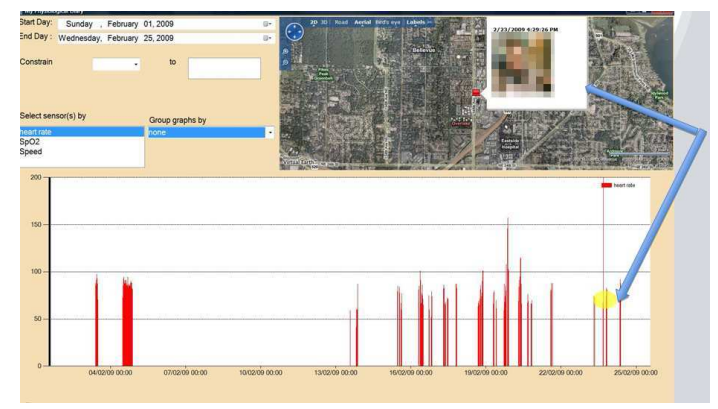

Figure 7: Anecdotal result - location + image + time context helping explain activities

\section{FUTURE WORK}

\subsection{Proposed Open Source Release}

It is our intention to make an open source release of the applications developed in this paper. This release will include the following:

- The cell phone logging application to run on Windows Mobile cell phones which accepts input from sensors transmitting data via Bluetooth

- A 2008 SQL Server Express database to store the physiological readings on an individual's desktop machine. Also included will be a small windows forms application to upload data from the cell phone XML sensor $\log$ file to the database

- The UI to help individuals effectively review their physiological activities. This interface automatically visualises newly added sensor values (after the developer has made minor adjustments to the cell phone logger, database, and database upload applications).

We believe an open source release of this code would be of benefit to the scientific community in general, but of particular significance to three groups:

- sensor device researchers who can now concentrate on the their hardware/chemistry/physics strengths instead of developing this system themselves

- machine learning researchers will be able to easily aggregate data to apply their techniques

- Athletes or health-conscious individuals can more easily make sense of the data they've been collecting

\subsection{Security}

Given the intensely private nature of physiological and medical data, a challenge for our community to deal with will be that of reassuring customers that their data will be stored and transmitted securely. Data must be securely transmitted from the physiological sensors to the individual's cell phone (i.e. surrounding people should not be able to $\log$ this data). Also data transmission to the physiological sensors must be secure too, e.g. Halperin et. al. have discovered that heart pacemakers can be wirelessly reset [12]! A very important consideration is how and where the data should be stored. We believe that in the long term it will be optimal to store this information in the cloud as this will allow people to access their data no matter where they areUin their homes, on the move, or possibly even when sitting down with their medical practitioner. Platforms such as Microsoft Health Vault and Google Health will play an increasingly important role in the near future.

\subsection{Symptom Detection}

In future we believe that it will be fruitful if our applications can make automatic recommendations to individuals on whether they may be displaying some symptoms of a given condition, e.g. such as monitoring one's heart beats over a long period of time to predict if they may be displaying symptoms of cardiovascular disease [14]. This may reduce the burden on health care professionals in future, as only people who display symptoms of conditions will go forward to seek further professional advice or treatment. To achieve such an aim, many years of research on large datasets will be necessary, so as to investigate the optimum machine learning algorithm(s) to detect possible symptoms of medical conditions, based on the lifelogged physiological signals as recorded by our applications. Indeed in the visual lifelogging domain researchers are already applying machine learning techniques to monitor the lifestyles of individuals [7]

\subsection{ZigBee}

Currently the sensors in our project communicate with the cell phone logger via the Bluetooth wireless transmission protocol. Our reason for this choice is that Bluetooth comes as standard on practically all modern cell phones, and as such we wanted to exploit this ubiquity. There is an unfortunate trade-off in the fact that Bluetooth is a relatively high-powered transmission protocol and has a limited number of simultaneous connections. A potential alternative in future may be a newly proposed wireless protocol named ZigBee, which has lower data transmission rates than Bluetooth, but is much less power consumptive, which may be ideal for a cell phone logger collecting data from wearable physiological sensors $[15,16,4]$

\section{CONCLUSIONS}

Early in this paper we have shown the potential benefits of lifelogging and gathering large amounts of physiological data. In the past much effort has focused on miniaturising hardware and sensors to facilitate the capture of large amounts of such data, and it is only recently that the community has seriously started tackling the problem of effectively querying this data.

In this work we contribute an open-source framework that can be run on Windows Mobile cell phones which enables the gathering of physiological data from multiple Bluetooth sensors. This release also includes an interface to effectively review this gathered data.

Indeed we exploit various aspects of the human memory system to facilitate users in quickly finding events of interest 
and in providing helpful contextual cues to explain the physiological values associated with these events. In this work we discuss anecdotal results based on data gathered by a single individual, but in future we intend to more thoroughly evaluate our techniques on a greater variety of individuals.

We also highlight a number of challenges the community faces before physiological lifelogging technologies begin to positively impact a large number of people, by individualising medical baselines and eventually making healthcare become proactive rather than reactive.

Acknowledgements: Authors AD and AS are supported by Science Foundation Ireland under grant 07/CE/I1147.

\section{REFERENCES}

[1] J. R. Anderson, editor. Language, Memory, and Thought. Lawrence Erlbaum Associates, 1974.

[2] R. Atkinson and R. Shiffrin. Human memory: A proposed system and its control processes. The psychology of learning and motivation, 2:89-195, 1968.

[3] A. Baddeley, editor. Your Memory: A User's Guide. Carlton Books, 2004.

[4] N. Baker. Zigbee and bluetooth strengths and weaknesses for industrial applications. Computing $\&$ Control Engineering Journal, 16:20-25, May 2005.

[5] D. Barreau, A. Crystal, J. Greenberg, A. Sharma, M. Conway, J. Oberlin, M. Shoffner, and S. Seiberling. Augmenting memory for student learning: Designing a context-aware capture system for biology education. Proceedings of the American Society for Information Science and Technology, 43:251-251, Oct 2007.

[6] E. Berry, A. Hampshire, J. Rowe, S. Hodges, N. Kapur, P. Watson, G. B. G. Smyth, K. Wood, and A. M. Owen. The neural basis of effective memory therapy in a patient with limbic encephalitis. Neurology, Neurosurgery, and Psychiatry with Practical Neurology, 80(3):582-601, 2009.

[7] D. Byrne, A. R. Doherty, C. G. Snoek, G. J. Jones, and A. F. Smeaton. Validating the detection of everyday concepts in visual lifelogs. In SAMT 2008 3rd International Conference on Semantic and Digital Media Technologies, pages 15-30, Berlin / Heidelberg, 2008. Springer.

[8] A. Doherty and A. F. Smeaton.. Utilising wearable sensor technology to provide effective memory cues. ERCIM News, No. 76, 2009.

[9] A. R. Doherty, C. O. Conaire, M. Blighe, A. F. Smeaton, and N. E. O'Connor. Combining image descriptors to effectively retrieve events from visual lifelogs. In MIR '08: Proceeding of the 1st ACM international conference on Multimedia information retrieval, pages 10-17, New York, NY, USA, 2008. ACM.

[10] D. Elsweiler. Supporting Human Memory in Personal Information Management. PhD thesis, Department of Computer and Information Sciences, University of Strathclyde, 2007.

[11] B. S. I. Group. Bluetooth technology chosen as health device standard. press release, Jun 2009.

[12] D. Halperin, T. S. Heydt-Benjamin, B. Ransford, S. S. Clark, B. Defend, W. Morgan, K. Fu, T. Kohno, and W. H. Maisel. Pacemakers and implantable cardiac defibrillators: Software radio attacks and zero-power defenses. In Symposium on Security and Privacy, pages 129-142. IEEE, 2008.

[13] S. Hodges, L. Williams, E. Berry, S. Izadi, J. Srinivasan, A. Butler, G. Smyth, N. Kapur, and K. Wood. Sensecamă: A retrospective memory aid. In UbiComp: 8th International Conference on Ubiquitous Computing, volume 4602 of $L N C S$, pages 177-193, Berlin, Heidelberg, 2006. Springer.

[14] Z. Jin, Y. Sun, and A. C. Cheng. Predicting cardiovascular disease from real-time electrocardiographic monitoring: An adaptive machine learning approach on a cell phone. In $E M B C-31^{\text {st }}$ International Conference of the IEEE Engineering in Medicine and Biology Society. IEEE, 2009.

[15] E. Jovanov, A. Milenkovic, C. Otto, and P. de Groen. A wireless body area network of intelligent motion sensors for computer assisted physical rehabilitation. Journal of NeuroEngineering and Rehabilitation, 2(1):6, 2005.

[16] E. Jovanov and Y. T. Zhang. Introduction to the special section on $\mathrm{m}$-health: Beyone seamless mobility and global wireless health-care connectivity. IEEE Transactions on Information Technology in Biomedicine, 8:405-414, December 2004.

[17] S. F. Larsen, C. P. Thompson, and T. Hansen. Remembering Our Past: Studies in Autobiographical Memory, chapter Time in Autobiographical Memory, pages 129-156. Cambridge University Press, 1996.

[18] Y.-H. Lin, I.-C. Jan, P. C.-I. Ko, Y.-Y. Chen, J.-M. Wong, and G.-J. Jan. A wireless pda-based physiological monitoring system for patient transport. IEEE Transactions on Information Technology in Biomedicine, 8:439-447, December 2004.

[19] A. Milenkovic, C. Otto, and E. Jovanov. Wireless sensor networks for personal health monitoring: Issues and an implementation. Computer Communications, 29(13):2521-2533, 2006.

[20] M. Montague and J. A. Aslam. Relevance score normalization for metasearch. In CIKM '01: Proceedings of the tenth international conference on Information and knowledge management, pages 427-433, New York, NY, USA, 2001. ACM Press.

[21] N. Oliver and F. F. Mangas. Healthgear: A real-time wearable system for monitoring and analyzing physiological signals. In International Workshop on Wearable and Implantable Body Sensor Networks, pages 1-4. IEEE, 2006.

[22] J. E. Purdy, M. R. Markham, B. L. Schwartz, and W. C. G. (2nd edition), editors. Learning and Memory. Thomson Learning, 2001.

[23] M. F. A. Rasid and B. Woodward. Bluetooth telemedicine processor for multichannel biomedical signal transmission via mobile cellular networks. IEEE Transactions on Information Technology in Biomedicine, 9:35-43, March 2005.

[24] D. L. Schacter. The Seven Sins of Memory: How the Mind Forgets and Remembers. Houghton Mifflin, 2001.

[25] M. Schwaibold, M. Gmelin, G. von Wagner, J. Schöchlin, and A. Bolz. Key factors for personal health monitoring and diagnosis device. In Mobile Computing in Medicine, Second Conference on Mobile Computing in Medicine, Workshop of the Project 
Group MoCoMed, GMDS-Fachbereich Medizinische Informatik \& GI-Fachausschuss 4.7, pages 143-150. GI, 2002.

[26] E. Tulving. Basic mechanisms in cognition and language, chapter Neurocognitive processes of human memory, pages 261-281. Elsevier, 1998.

[27] I. T. Union. Worldwide mobile cellular subscribers to reach 4 billion mark late 2008. press release, Sep 2008. 